Belo Horizonte, v. 13, n. 3, p. 121-139, set.-dez. 2020 - ISSN 1983-3652 DOI : $10.35699 / 1983-3652.2020 .25107$

\title{
EFECTOS SOBRE LA METODOLOGÍA FLIPPED CLASSROOM A TRAVÉS DE BLACKBOARD SOBRE LAS ACTITUDES HACIA LA ESTADÍSTICA DE ESTUDIANTES DEL GRADO DE EDUCACIÓN PRIMARIA: UN ESTUDIO CON ANOVA MIXTO
}

EFFECTS ON THE FLIPPED CLASSROOM METHODOLOGY THROUGH BLACKBOARD ON THE ATTITUDES TOWARDS STATISTICS OF STUDENTS OF THE PRIMARY EDUCATION DEGREE: A STUDY WITH MIXED ANOVA

\author{
EFEITOS DA METODOLOGIA SALA DE AULA INVERTIDA POR MEIO DO \\ BLACKBOARD NAS ATITUDES EM RELAÇÃO ÀS ESTATÍSTICAS DE ESTUDANTES \\ DO ENSINO FUNDAMENTAL: UM ESTUDO COM ANOVA MISTA
}

\author{
Francisco David Guillén Gámez \\ Universidad de Almería, Espanha \\ dguillen@ual.es \\ Ernesto Colomo Magaña \\ Universidad de Málaga, Espanha \\ ecolomomagana@gmail.com \\ Enrique Sánchez Rivas \\ Universidad de Málaga, Espanha \\ enriquesr@uma.es \\ Rocío Pérez del Río \\ Universidad de Málaga, Espanha \\ rperezdelrio@uma.es
}

RESUMEN: Este estudio evalúa si el uso de la metodología Flipped Classroom apoyado por explicaciones del profesorado a través de plataformas virtuales de aprendizaje (Blackboard) produce una alteración en las actitudes hacia la estadística de futuros docentes de Educación primaria, en función del sexo. Para ello, se utilizó un diseño cuasiexperimental (pre-test / post-test) y como técnica estadística ANOVA mixto con tres medidas repetidas. La muestra fue de 66 estudiantes de la Facultad de Educación de la Universidad de Almería (UAL). Para realizar el análisis comparativo en las actitudes hacia la estadística, fue utilizado el instrumento CAHE creado por Camacho et al. (2016) compuesto por un total de 16 ítems, clasificado en tres dimensiones: emocional negativa (temor, miedo, tensión hacia la estadística), emocional positiva (gusto, agrado, diversión hacia la estadística), y utilidad hacia la estadística. Los resultados evidenciaron diferencias estadísticamente significativas entre las actitudes del alumnado entre el pretest y el post-test al utilizar dicha metodología, pero no la interacción con el sexo. Los resultados ponen de relieve que el uso de la metodología Flipped Classroom puede ayudar a mejorar las actitudes hacia la investigación estadística, siendo un arma imprescindible para cualquier maestro que quiere innovar en sus clases.

PALABRAS CLAVE: Estudiantes. Futuros maestros. Educación. Estadística. e-Learning. Flipped Classroom. 
ABSTRACT: This study assesses whether the use of the Flipped Classroom methodology supported by explanations by teachers through a Learning management system (Blackboard) produces an alteration in the attitudes towards statistics of future teachers of Primary education, with gender interaction. For this, a quasi-experimental design (pretest / post-test) was used, and as a statistical technique a mixed ANOVA with three repeated measures. The sample was 66 students from the Faculty of Education of the University of Almería (UAL). To carry out the comparative analysis of attitudes towards statistics, the CAHE instrument created by Camacho et al. (2016) made up of a total of 16 items, classified into three dimensions: negative emotional (fear, fear, tension towards statistics), positive emotional (liking, liking, fun towards statistics), and utility towards statistics. The results showed statistically significant differences between the students' attitudes between the pre-test and the post-test, but not the interaction with gender. The results highlight that the use of the Flipped Classroom methodology can help improve attitudes towards statistical research, being an essential weapon for any teacher who wants to innovate in their classes.

KEYWORDS: Students. Future teachers. Education. Statistics. E-Learning. Flipped Classroom.

RESUMO: Este estudo avalia se a utilização da metodologia sala de aula invertida apoiada em explicações dos professores por meio de plataformas virtuais de aprendizagem (Blackboard) produz uma alteração nas atitudes em relação às estatísticas dos futuros professores do ensino fundamental, dependendo do gênero. Para isso, foi utilizado um desenho quase experimental (pré-teste/pós-teste) e como técnica estatística mista ANOVA com três medidas repetidas. A amostra foi composta por 66 alunos da Faculdade de Educação da Universidade de Almería (UAL). Para realizar a análise comparativa das atitudes em relação à estatística, foi utilizado o instrumento CAHE criado por Camacho et al. (2016) composto por um total de 16 itens, classificados em três dimensões: emocional negativo (medo, medo, tensão com as estatísticas), emocional positivo (gostar, gostar, divertimento com as estatísticas) e utilidade para as estatísticas. Os resultados mostraram diferenças estatisticamente significativas entre as atitudes dos alunos entre o pré-teste e o pós-teste com a utilização dessa metodologia, mas não a interação com o sexo. Os resultados destacam que a utilização da metodologia sala de aula invertida pode ajudar a melhorar as atitudes em relação à pesquisa estatística, sendo uma arma essencial para qualquer professor que queira inovar nas suas aulas.

PALAVRAS-CHAVE: Estudantes. Futuros professores. Educação. Estatísticas. eLearning. Sala de aula invertida.

\section{Introducción}

La Estadística es una materia habitual en los planes de estudio universitarios de diferentes áreas de conocimientos, debido a su relevancia para analizar e interpretar datos (BLANCO, 2008), favoreciendo así un razonamiento crítico que se sustente en evidencias objetivas. Pese a su importancia, el aprendizaje de la misma se convierte en un problema entre el alumnado de educación superior (RODRÍGUEZ; MONTAÑEZ; ROJAS, 2010). Dentro del ámbito de las ciencias sociales y humanidades, donde se encuadra las disciplinas vinculadas a las ciencias de la educación, nos encontramos con 
la problemática de la existencia de diferentes niveles respecto a los conocimientos previos sobre estadística, pudiendo encontrar casos de estudiantes que carecen completamente de los mismos. El problema subyace cuando la mayoría del alumnado matriculado en los grados educativos de las múltiples Facultades de Educación existentes, han cursado el itinerario de humanidades en bachillerato, un factor que influye en poseer un menor bagaje de conocimientos más amplios sobre matemáticas y estadísticas, una asignatura que es clave para el desarrollo del profesional educativo (IMMEKUS, 2019).

Este hecho se traduce en la presencia de preconcepciones y actitudes negativas en el alumnado a la hora de cursar la materia de Estadística u otras que incluyan en su contenido referencias a la misma (CARMONA, 2004; KHAVENSON; OREL; TRYAKSHINA, 2012; MONDÉJAR; VARGAS; MONDÉJAR, 2007; TOMÁS; LIMA; PACHECO, 2015). A este respecto, las actitudes hacia la estadística son conductas, cogniciones y emociones caracterizadas por su intensidad y polaridad (RODRÍGUEZ, 2011), y por su relación con otros elementos de la materia (como el docente, la propuesta metodológica, la carga de trabajo, etc.), que influyen tanto en el proceso como en el rendimiento educativo, pudiendo determinar el éxito o fracaso respecto al mismo (RAMÍREZ; SCHAU; EMMIOGLU, 2012; ROSLI; MAAT; ROSLI, 2017). Pueden surgir como fruto de las creencias personales, de experiencias previas o de transponer las actitudes del alumnado hacia las matemáticas por la relación de esta materia con la Estadística. Son un aspecto a considerar en todos los procesos formativos, ya que pueden convertirse en un factor predictivo del rendimiento académico.

El estudio de los aspectos actitudinales hacia el aprendizaje de la estadística ha generado un ámbito específico de estudio, donde se han llevado a cabo investigaciones en estudiantes de etapas secundaria (BOND; PERKINS; RAMÍREZ, 2012), preuniversitaria (CASAS et al. 2018; SALINAS; MAYÉN, 2016) y universitaria (PÉREZ et al., 2015), focalizando estos en titulaciones como psicología (COMAS et al., 2017; ESCALANTE; REPETTO; MATTINELLO, 2012), medicina (PEÑA et al., 2015) o ciencias de la educación (APARICIO et al., 2015; HANNIGAN; GILL; LEAVY, 2013; LEÓN et al., 2020). En nuestro caso, situaremos el foco en el área de educación y en las actitudes de los estudiantes ante el proceso de aprendizaje de la Estadística, un fenómeno que viene siendo investigado en España (ESTRADA; BAZÁN; APARICIO, 2013; RUIZ DE MIGUEL, 2015). En este sentido, estudios como el de Vilà y Rubio (2016), con 203 estudiantes de pedagogía de la Universidad de Barcelona, distinguieron tres perfiles según las actitudes del alumnado hacia la estadística: actitud negativa, caracterizados por el bajo nivel de conocimientos respecto a la estadística y las habilidades numéricas; actitud ansiosa, fruto del miedo suscitado por la materia; y actitud positiva, sustentada en su gusto por la asignatura. Por su parte, el trabajo de Ordóñez, Romero y Ruiz (2019), con 855 alumnos de la facultad de educación de la Universidad Complutense, distinguió únicamente dos perfiles de estudiantes: el negativo, con actitudes desfavorables y baja consideración de la utilidad de la estadística; y el positivo, con actitudes favorables y alta consideración de su utilidad.

De este modo, la existencia de una predisposición negativa en actitudes supone un obstáculo tanto para la labor del docente como la del alumnado, debido a que el proceso educativo se desarrolla en un contexto de desmotivación, desinterés y angustia. Una situación que puede empeorarse cuando las metodologías y estrategias de aprendizaje de la Estadística se sustentan, en exclusiva, en el razonamiento matemático en lugar de orientarse a un raciocinio estadístico sobre elementos de interés académico para el 
alumnado (TISHKOVSKAYA; LANCASTER, 2012), empeorando así el clima de clase y dificultando el proceso de andamiaje. Todo ello dificulta la adquisición de los conocimientos y la correspondiente consecución de los objetivos académicos estipulados (VANHOOF et al., 2011), manteniendo esa predisposición negativa hacia su utilidad y aplicación incluso tras haber superado la materia.

El análisis de las actitudes en estadística tiene ya una cierta tradición, encontrándose una gran variedad de investigaciones en las últimas décadas, coincidiendo casi todas en el análisis del sexo (HARVEY, 1985; ANASTASIADOU, 2005). Si nos acercamos a la última década, Pérez et al. (2015) analizó las actitudes hacia la estadística de 545 estudiantes colombianos de una universidad privada de Bogotá, encontrando que no había diferencias significativas respecto al sexo; resultados similares fueron encontrados por Estrada, Bernabeu y Aymemí (2004), Roca (2008), Chiesi y Primi (2017). Sin embargo, algunos estudios han encontrado que los hombres tiene actitudes más positivas hacia la estadística en comparación con las mujeres (PAPANASTASIOU; ZEMBYLAS, 2008; VAHEDI; FARROKHI; BEVRANI, 2011; KOH; ZAWI, 2014; CHOWDHURY, 2018). Sin embargo, otros investigadores tuvieron hallazgos diferentes como por ejemplo Elizar y Darmawan (2018) encontrando que la actitud positiva de las mujeres con respecto a las matemáticas es mayor en comparación con los hombres.

Partiendo de dichas barreras y de la existencia de diferentes perfiles de alumnado, en lo que respecta a su predisposición y conocimientos hacia esta materia, la idea es promover propuestas de enseñanza-aprendizaje que sean capaces de adaptarse a las necesidades, requerimientos e intereses de los diferentes grupos. Se trata de lograr cambiar las actitudes negativas y la desmotivación hacia la Estadística en una disposición positiva hacia dicha disciplina (DARIAS, 2000), debido al vínculo significativo existente, en esta materia, entre actitudes positivas y logro académico (GUÀRDIA et al., 2006). En este sentido, estudios como el de Arteaga y García (2010) lograron resultados positivos en procesos de enseñanza adaptativa sobre asignatura con contenidos de matemáticas. No olvidemos que uno de los cometidos docentes es, desde la labor pedagógica, diseñar e implementar propuestas formativas que sean motivadoras para el desarrollo de la asignatura de Estadística. Por tanto, una alternativa es la apuesta por convertir al alumnado en el protagonista del proceso educativo a través de la implementación de metodologías activas de aprendizaje. Una propuesta avalada por investigaciones como la de Froelich, Stephenson y Duckworth (2008), quienes evidenciaron mejoras en las actitudes y resultados de su alumnado al implementar metodologías orientadas a la práctica y la participación. Se trata de que el discente asuma una mayor responsabilidad en su aprendizaje, trasladando el protagonismo del proceso desde el docente al estudiante, en un ambiente didáctico más lúdico y experiencial.

Dentro de la variedad de metodologías activas, este trabajo se desarrolló mediante la aplicación del Flipped Classroom o aula invertida. Estamos ante una metodología que, siguiendo a Sánchez, Ruiz y Sánchez (2017) ha recibido diferentes denominaciones como clase al revés, instrucción inversa o enseñanza invertida. A nivel conceptual, consiste en trabajar los contenidos fuera de clase, haciendo uso de diferentes recursos y materiales mediante las tecnologías de la información y la comunicación (vídeos, lecturas, presentaciones online, entre otros), diseñados o facilitados por el docente, de forma que el tiempo de clase se use para solventar dudas, debatir, profundizar en lo trabajado y plantear problemas para su resolución, aplicando de forma significativa los contenidos ya trabajados (MOTOS et al., 2019). Bishop y Verleger (2013, p. 5), sintetiza su explicación 
en "realizar las actividades de aprendizaje interactivas en grupo dentro de la clase, y la instrucción individual a través del ordenador fuera de la misma". De este modo, el estudiante puede gestionar mejor el aprendizaje de los contenidos, al tener materiales con los que puede preparar la sesión de clase, a la par que la enseñanza en el aula se transforma en una realidad mucho más práctica, significativa e innovadora, dando lugar al desarrollo de la creatividad, la reflexión o las habilidades sociales (MARTíN; TOURÓN, 2017; SOSA; PALAU, 2018). Por tanto, se trata de una estrategia pedagógica que conlleva replantear tanto los procesos como los tiempos formativos, dando mayor peso a competencias como aprender a aprender (antes de la sesión presencial) y aprender a hacer (durante la sesión presencial).

Existen cuatro pilares fundamentales a considerar en el modelo Flipped (LITTLE, 2015; SÁNCHEZ; SÁNCHEZ; RUIZ, 2019):

- Flexibilidad: tanto en el aprendizaje (con diferentes entornos y posibilidades de elección para el alumnado sin barreras físicas ni temporales) como en la labor docente (organización, tipología de actividades, evaluación, etc.).

- Cultura de aprendizaje: El alumnado pasa de un rol pasivo (docente como fuente primaria de información) a un papel activo (docente guía el proceso y el estudiante es protagonista en la construcción del conocimiento).

- Contenido intencional: seleccionados o diseñados en función de los fines didácticos perseguidos, siendo labor del docente estipular cuáles serán trabajados de forma autónoma por el alumnado y cuáles se implementarán de forma práctica en el aula.

- Educador profesional: la labor del docente va más allá de la transmisión de conocimientos, siendo clave su labor didáctica y pedagógica, aplicando los cambios, adaptaciones, innovaciones o retos que sean precisos para mejorar la labor formativa, sustentando sus decisiones en un proceso reflexivo y autocrítico de su labor.

Esta metodología ha sido implementada en multitud de experiencias, siendo objeto de múltiples investigaciones (GALINDO; BEZANILLA, 2019; KONG, 2014; LÓPEZ; POZO; DEL PINO, 2019; MENDAÑA; POY; LÓPEZ, 2019). Entre las razones, encontramos diferentes ventajas y beneficios que reporta su implementación, los cuales recogemos a continuación:

- Permite disponer de un mayor tiempo para personalizar la atención respecto a las dificultades de los estudiantes e incrementar los feedbacks durante las sesiones de clase (MCBRIDE, 2015; TOURÓN; SANTIAGO, 2015), ya que el tiempo de clase no se dedica a la explicación de unos contenidos que ya están a disposición del alumnado, aumentándose así el número de interacciones entre docente-alumno en el aula.

- Favorece el respeto a los diferentes ritmos de aprendizaje, permitiendo al alumnado ser más autónomo e independiente (O'FLAHERTY; PHILIPHS, 2015; LIMNIOU; SCHERMBRUCKER; LYONS, 2018), pues se convierten en responsables de trabajar los materiales propuestos sobre los contenidos teóricos sin los cuales no sería factible el desarrollo de este modelo.

- Se implementa un mayor número de actividades prácticas en el aula, ya que los contenidos teóricos son trabajados fuera de clase, favoreciendo así la relación entre teoría-practica de forma significativa (MASON; SHUMAN; COOK, 2013). 
- Se tienen en consideración los diferentes estilos de aprendizaje, pudiendo presentar los contenidos para trabajar fuera del aula en los formatos que más se ajusten y beneficien a su alumnado (COTO, 2020).

- Optimización del tiempo, permitiendo no solo abarcar los contenidos previstos, sino profundizar en temas o cuestiones de interés vinculadas a la materia (GONZÁLEZ et al., 2016), fomentando así aspectos como la curiosidad, la motivación o las competencias para investigar.

- Mejora explícita de los procesos de evaluación, ya que el docente ha podido realizar un seguimiento más personalizado y se han generado un mayor y variado número de evidencias sobre los aprendizajes, favoreciendo mayor objetividad al llevar a cabo procesos de triangulación.

Sin embargo, también podemos encontrar dificultades asociadas a la implementación de este modelo, como es el compromiso del estudiante. En este sentido, el ubicar al estudiante en el centro del proceso de aprendizaje, invirtiendo los roles tradicionales del aula, conlleva un alto nivel de compromiso y esfuerzo, de manera que, si este no es real, no es factible un buen desarrollo del proceso de enseñanza-aprendizaje con esta estrategia metodológica (JOHNSON, 2013; STRAYER, 2012). Partiendo de sus pros y sus contras, son varias las experiencias en las que el desarrollo de asignaturas 0 cursos de estadística en el ámbito universitario, dentro de diferentes áreas, se han desarrollado con una metodología activa como el Flipped Classroom (CARLSON; WINQUIST, 2011; METAUTE et al., 2018; NIELSEN; BEAN; LARSEN, 2018; TAWFIK; LILLY, 2015; SCHWARTZ 2014; SHINABERGER, 2017; TRIANTAFYLLOU; TIMCENKO, 2014).

El apostar por esta metodología encuentra su fundamentación en las diferentes investigaciones que subrayan la eficacia del Flipped Classroom. Algunos de los estudios han aplicado pre-test/post-test, donde la evolución de los resultados, en diferentes momentos, ha evidenciado la eficacia de implementar esta metodología (AYCICEK; YELKEN, 2018; CRONHJORT; FILIPSSON; WEURLANDER, 2018; ESPERANZA; FABIAN; TOTO, 2016; SENGER, 2016). Otros trabajos han analizado la eficacia enfocándose en la mejora del rendimiento del alumnado (calificaciones) al implementar el Flipped Classroom, realizando trabajos con grupos de control y experimentales, como los casos de Peterson (2016), Touchton (2015) y Winquist y Carlson (2014). Cabe destacar el trabajo de Wilson (2013), por la utilización de pre-test y post-test en la implementación de una metodología Flipped Classroom para un curso de estadística, logrando mejorar tanto las actitudes del alumnado hacia la asignatura como sus resultados.

Siguiendo esta misma línea, el propósito de este estudio es conocer la eficacia de la metodología Flipped Classroom apoyado por la plataforma virtual Blackboard sobre las actitudes hacia la estadística del alumnado del Grado de Educación Primaria. En concreto, los objetivos son: 1) conocer la eficacia de esta metodología utilizando un diseño pre-test y post-test con un diseño mixto de medidas repetidas (antes, durante y después); y 2) analizar las actitudes hacia la estadística del alumnado en función del sexo.

Bajo esta premisa, se establecen dos hipótesis estadísticas nulas del estudio:

- El alumnado no mejora significativamente su actitud hacia la estadística después de aplicar la metodología Flipped Classroom

- No se encontraron diferencias significativas en las actitudes hacia la estadística en 
función del sexo en los diferentes momentos en que se aplica la metodología Flipped Classroom.

\section{Método}

\subsection{Diseño}

Un estudio cuantitativo ex post facto y cuasiexperimental con medidas pre-test y post-test ha sido llevado a cabo. Ex post facto ya que se ha pretendido recolectar las actitudes hacia la estadística de los participantes a partir de un instrumento de medida, y cuasiexperimental no equivalente ya que los grupos no eran aleatorios, sino que estaban ya establecidos. La recogida de los datos fue a través de un cuestionario compuesto por tres momentos, antes de comenzar la metodología Flipped Classroom (pretest), durante el desarrollo del curso (post-test 1) y al finalizar la metodología (post-test 2). Una vez recolectado los datos de los participantes en ambos momentos, análisis descriptivos e inferenciales fueron llevados a cabo para conocer la eficacia de la metodología Flipped Classroom a través de Blackboard.

\subsection{Participantes}

Para medir la eficacia de la metodología, un muestro no probabilístico fue utilizado de manera intencionada con 66 estudiantes del Grado de Educación Primaria de la Universidad de Almería (UAL) en el curso académico 2019/2020. Respecto al sexo, el $45.5 \%(n=30)$ eran hombres, mientras que el $54.5 \%(n=36)$ eran mujeres. La edad media en conjunto fue de 19.41 años con una desviación típica de 2.54.

\subsection{Instrumento}

Con el fin de medir las actitudes hacia la estadística de los alumnos, fue utilizado el instrumento CAHE (cuestionario de actitudes hacia la estadística) elaborado por Camacho et al. (2016). El instrumento está compuesto por 16 ítems y 3 dimensiones: la dimensión se denomina emociones positivas, y está enfocado en las actitudes hacia la estadística a las cuales al alumnado le producen temor, miedo o tensión este tipo de aprendizaje, y conta de 8 ítems (por ejemplo el ítem EAE2 "La asignatura de estadística se me da bastante mal"); la segunda dimensión se llama emocional positiva, enfocándose en el gusto, agrado o diversión que el alumnado puede sentir al estar aprendiendo este tipo de contenidos, y consta de 4 ítems (por ejemplo el ítem EAE4 "Utilizar la estadística es una diversión para mi"); y por último, la dimensión de utilizada, enfocada en las actitudes del alumnado sobre su percepción de lo útil que puede llegar a ser el aprendizaje y puesta en práctica en contextos reales y significativos de este tipo de aprendizaje, compuesto por 4 ítems (por ejemplo el ítem EAE11 "Saber utilizar la estadística incrementaría mis posibilidades de trabajo"). Para medir las actitudes fue utilizado una escala Likert de cinco puntos, donde el valor 1 se asimilaba a la variable cualitativa "totalmente en desacuerdo" y el valor 5 a la variable "totalmente de acuerdo".

El instrumento presentaba las suficientes evidencias para ser válido y fiable ya que cumplía con las correctas propiedades psicométricas. Respecto a la fiabilidad, el instrumento recogía un alfa de Cronbach muy satisfactorio, $\alpha=0.902$. Específicamente, 
cada una de las dimensiones también presentaron niveles satisfactorios, dimensión emociones positivas, $\alpha=0.92$; dimensión emocional positiva, $\alpha=0.774$; dimensión utilidad, $\alpha=0.745$.

Respecto a la validez, el análisis factorial exploratorio (AFE) explicó un $62.78 \%$ de la varianza total de las puntuaciones verdaderas del instrumento, siendo del $33.48 \&$ para la primera dimensión, $15.94 \%$ para la segunda, y $13.35 \%$ para la tercera. Respecto al análisis factorial confirmatorio (AFC), la estructura interna del test presentó un buen ajuste $\left(\mathrm{X}^{2}=123.20 ; 101 \mathrm{gl} ; \mathrm{p}=.066\right.$, RMSEA=.024; $\left.\mathrm{CFI}=\mathrm{NNFI}=1 ; \mathrm{SRMR}=.045\right)$ y saturaciones significativas.

\subsection{Procedimiento}

Para el presente estudio, ha sido utilizado 37.5 horas teóricas y prácticas de la asignatura Investigación Educativa en Educación Primaria (IEEP) del primer curso del Grado de Educación Primaria de la Universidad de Almería (UAL) durante el segundo cuatrimestre del curso académico 2019/2020. En la Tabla 1 aparecen los principales objetivos y contenidos de la asignatura relacionados específicamente con la metodología cuantitativa según recoge la guía docente. Además de la puesta en práctica de esta metodología, se ha utilizado la plataforma virtual Blackboard, tanto para la enseñanza de los contenidos teóricos, como para las prácticas.

Tabla 1: Breve descripción de objetivos y contenidos de la asignatura IEEP.

\begin{tabular}{|c|c|}
\hline Objetivos & Contenidos \\
\hline $\begin{array}{l}\text { - Conocer las características básicas de los planteamientos } \\
\text { cuantitativos en investigación educativa. - Distinguir entre la } \\
\text { descripción, el análisis y la interpretación de los datos } \\
\text { estadísticos a lo largo de una investigación. - Saber diseñar } \\
\text { un proyecto de investigación educativa - Saber utilizar } \\
\text { algunas de las técnicas de producción de datos más } \\
\text { usuales en investigación educativa. }\end{array}$ & $\begin{array}{l}\text { - Concepto de variable: Tipos. - Diseño } \\
\text { experimental y no experimental. - } \\
\text { Investigación correlacional. - Técnicas } \\
\text { paramétricas y no paramétricas. - Validez y } \\
\text { fiabilidad de los instrumentos. - Ejemplos } \\
\text { de técnicas para analizar cuestionarios. }\end{array}$ \\
\hline
\end{tabular}

Fuente: de los autores.

La metodología utilizada consistió en el visionado de las lecciones teóricas y prácticas con antelación a las clases virtuales, con el propósito no sólo de tener más tiempo para realizar tareas con SPSS y resolver dudas en las técnicas estadísticas utilizadas, sino también para fomentar la capacidad de estudio y aprender nuevos contenidos de manera autónoma. Después, en las claves virtuales, el profesor descubre las dudas y dificultades del alumnado, reforzando lo aprendido hasta el momento con casos prácticos.

La plataforma Blackboard fue el soporte técnico sirviendo no solo para el repositorio de los recursos y creación de glosarios, sino para crear una comunicación tanto asíncrona para que los estudiantes planteen y resuelvan sus dudas a través de foros, como síncrona a través del propio sistema de videoconferencia donde tenían efecto las clases virtuales.

Para el visionado de las lecciones fueron utilizado tres tipos de materiales: por un 
lado, diferentes píldoras informativas extraídas de canales de YouTube enfocados en técnicas estadísticas, creadas por usuarios de la red; videos elaborados por el profesor de la asignatura con el planteamiento y resolución de casos prácticos; y por último, de ejemplos de artículos científicos en los cuáles se emplean las técnicas aprendidas con el fin de que aprender a presentar los resultados más importantes.

\subsection{Técnicas de análisis de los datos}

El análisis de los resultados, requirió de los siguientes procedimientos:

- En primer lugar, análisis descriptivos de cada una de las dimensiones fueron llevados a cabo en función del sexo del alumnado.

- En segundo lugar, para medir la eficacia de la metodología Flipped, un ANOVA mixto de medidas repetidas fue utilizado. En el factor intra-grupos se utilizó la variable dependiente actitudes hacia la estadística en tres momentos: antes del inicio de la metodología Flipped Classroom; durante el desarrollo del curso; y al finalizar el curso. Como factor entre-grupos se utilizó la variable nominal dicotómica sexo. Además, fueron llevado a cabo unos análisis exploratorios previos, con especial atención al cumplimiento de los supuestos de este tipo de técnicas: esfericidad de la varianza (Mauchly), corrección de grados de libertad por HuynhFeldt y comparaciones por pares con las pruebas de Bonferroni. El software utilizado fue SPSS V. 22.

\section{Resultados}

\subsection{Análisis descriptivos de la metodología Flipped Classroom}

La Tabla 2 muestra las estadísticas descriptivas para en tres momentos distintos del desarrollo de la metodología Flipped Classroom (antes, durante y después) sobre la actitud global hacia la estadística (puntuaciones totales del instrumento). La tabla incluye la media (m), la desviación típica (dt) y la prueba de normalidad Kolmogorov-Smirnov (KS).

Tabla 2: Resultados descriptivos y exploratorios.

\begin{tabular}{|c|c|c|c|c|c|c|}
\hline \multirow[t]{2}{*}{ Grupo } & \multirow{2}{*}{ Tiempo } & \multirow{2}{*}{$\mathrm{m}$} & \multirow{2}{*}{ dt } & \multicolumn{3}{|c|}{ Kolmogorov-Smirnov } \\
\hline & & & & $\mathrm{KS}$ & $g l$ & p. \\
\hline \multirow{3}{*}{ Antes } & Hombre & 2.65 & 0.52 & 0.096 & 30 & 0.200 \\
\hline & Mujer & 2.66 & 0.51 & 0.113 & 36 & 0.200 \\
\hline & Total & 2.65 & 0.51 & 0.075 & 66 & 0.200 \\
\hline \multirow{3}{*}{ Durante } & Hombre & 2.90 & 0.38 & 0.097 & 30 & 0.200 \\
\hline & Mujer & 2.73 & 0.34 & 0.118 & 36 & 0.200 \\
\hline & Total & 2.81 & 0.37 & 0.081 & 66 & 0.200 \\
\hline & Hombre & 3.10 & 0.35 & 0.149 & 30 & 0.087 \\
\hline \multirow[t]{2}{*}{ Después } & Mujer & 2.96 & 0.32 & 0.132 & 36 & 0.115 \\
\hline & Total & 3.03 & 0.34 & 0.106 & 66 & 0.064 \\
\hline
\end{tabular}

Fuente: de los autores. 
Respecto a la recolección de las actitudes antes de comenzar a trabajar con la metodología Flipped, se observa que el alumnado poseía unas creencias no realmente positivas $(M=2.65 \pm 0.51)$, siendo prácticamente iguales tanto en las mujeres $(M=2.66 \pm$ $0.51)$ como en los hombres $(M=2.65 \pm 0.51)$. Las actitudes alcanzadas por el alumnado una vez comenzado el curso fueron ligeramente más positivas $(\mathrm{M}=2.81 \pm 0.37)$, siendo más favorables en los hombres $(M=2.90 \pm 0.38)$ respecto a las mujeres $(M=2.73 \pm 0.34)$. Esta observación se corroboró en las actitudes encontradas al finalizar el curso $(\mathrm{M}=3.03 \pm$ $0.34)$, encontrándose creencias más positivas en los hombres $(M=3.10 \pm 0.35)$ frente a las mujeres $(M=2.96 \pm 0.32)$. Por último, el test de Kolmogorov-Smirnov asumió la normalidad de los datos en cada uno de los grupos en los tres momentos de medida (p. > 0.05).

\subsection{Supuestos previos de comprobación en ANOVA Mixto}

La normalidad de los datos se comprobó en la Tabla 1. Además, a través de la observación de los gráficos Q-Q normal y Q-Q sin tendencia se constata que las distribuciones se aproximan a la normalidad en cada uno de los subgrupos de la variable de medida. La prueba $M$ de Box $(M=11.484$; p. > 0.05) permitió aceptar la hipótesis de igualdad de matrices de varianzas-covarianzas y, por tanto, se concluye que los grupos son iguales. El test de Levene determinó que se cumplió el supuesto de homogeneidad de las varianzas, en los tres factores intra-sujetos: antes, $F(1,64)=0.001$, p. $=0.989$; durante, $F(1,64)=0.812$, p. $=0.371$; y después, $F(1,64)=0.967$, p. $=0.390$.

Por último, como se puede ver en la Tabla 3, el test de esfericidad de Mauchly fue significativo, no cumpliéndose el supuesto de esfericidad de la varianza. Por lo tanto, en adelante, se interpretarán las significancias calculadas con la corrección de grados de libertad (g.I.) de Huynh-Feldt.

Tabla 3: Prueba de esfericidad de Mauchly.

\begin{tabular}{|c|c|c|c|c|c|c|}
\hline \multirow{2}{*}{$\begin{array}{c}\text { W de } \\
\text { Mauchly }\end{array}$} & \multirow{2}{*}{$\begin{array}{c}\text { Aprox. Chi- } \\
\text { cuadrado }\end{array}$} & gl & Sig. & $\begin{array}{c}\text { Greenhouse- } \\
\text { Geisser }\end{array}$ & Huynh-Feldt & $\begin{array}{c}\text { Límite } \\
\text { inferior }\end{array}$ \\
\hline 0.073 & 164.700 & 2 & 0.001 & 0.519 & 0.528 & 0.500 \\
\hline
\end{tabular}

Fuente: de los autores.

\subsection{Análisis de la prueba ANOVA mixto}

Atendiendo a la corrección Huynh-feldt, los resultados del ANOVA mixto de medidas repetidas (Tabla 4), indicaron la existencia de efectos principales en el factor momentos de la metodología Flipped Classroom, $F(1.056,67.6054)=18.661$, p. $<0.005$. Sin embargo, no se hallaron efectos de interacción significativos entre el factor intragrupos y el factor entre-grupos (p. > 0.05). 
Tabla 4: Valoración de los efectos intra-grupos con la corrección de grados de libertad de Huynh-feldt.

\begin{tabular}{|l|c|c|c|c|c|c|}
\hline Origen & $\begin{array}{c}\text { Tipo III de suma } \\
\text { de cuadrados }\end{array}$ & gl & $\begin{array}{c}\text { Media } \\
\text { cuadrática }\end{array}$ & F & Sig. & $\begin{array}{c}\text { Eta parcial al } \\
\text { cuadrado }\end{array}$ \\
\hline Metodología Flipped & 4.727 & 1.056 & 4.475 & 18.661 & 0.001 & 0.226 \\
\hline Metodología FlippedSexo & 0.280 & 1.056 & 0.265 & 1.106 & 0.300 & 0.017 \\
\hline Error (Factor 1) & 16.211 & 67.605 & 0.240 & & & \\
\hline
\end{tabular}

Fuente: de los autores.

A modo resumen, se puede matizar que, aunque existen diferencias significativas en las actitudes del alumnado hacia la estadística entre los tres momentos de recogida de datos de la metodología Flipped (antes, durante y después), no tiene un efecto diferente según el sexo del alumnado. Estos resultados se pueden visualizar fácilmente en el Gráfico 1.

Gráfico 1: Actitud hacia la estadística del alumnado por sexo, acorde a cada momento del curso Flipped Classroom.

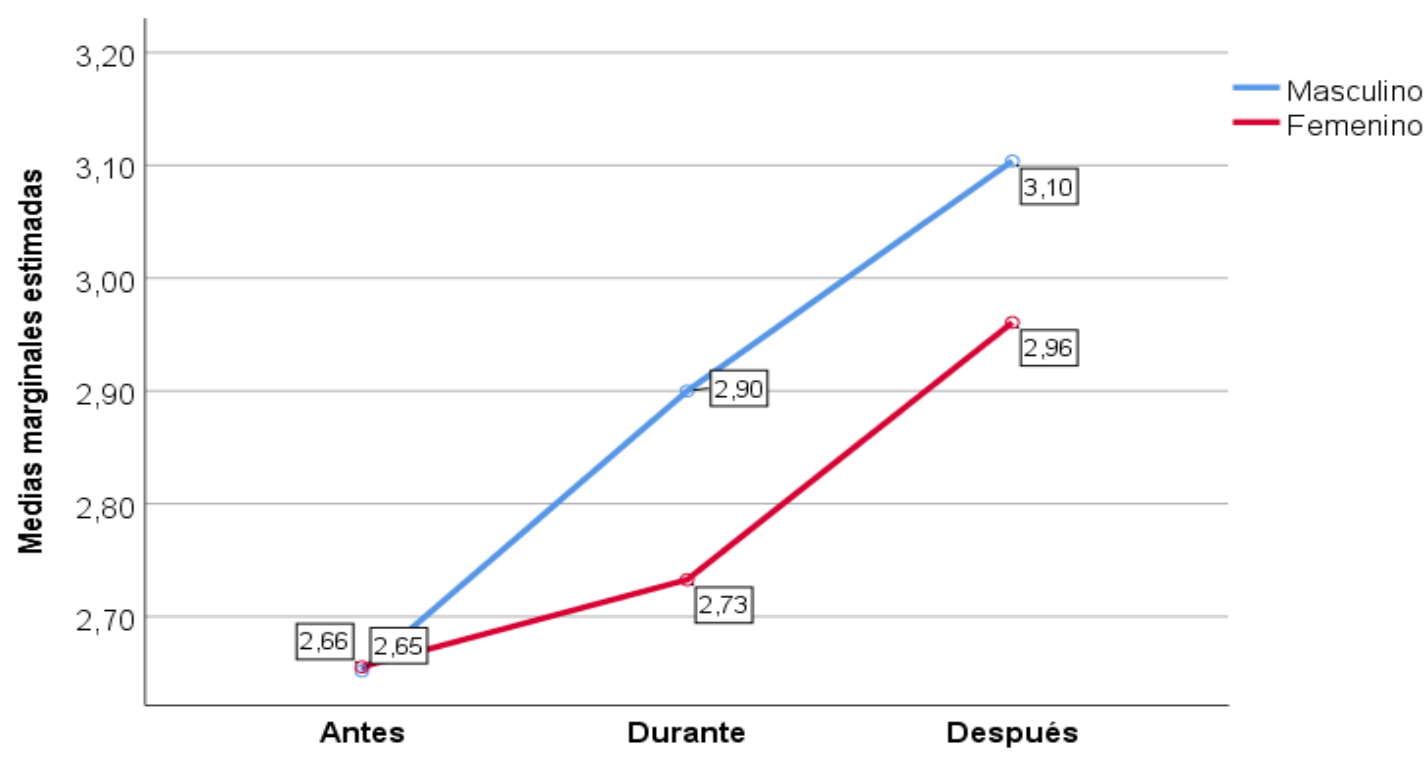

Fuente: de los autores.

Para determinar si existen diferencias significativas entre los tres momentos de la metodología Flipped Classroom, se hace una prueba de comparación por parejas, ajustando el error por el método de Bonferroni. El Gráfico 1 muestra que las diferencias de las actitudes del alumnado hacia la estadística se dan entre los momentos de la metodología más distantes u opuestos, es decir, entre antes de comenzar y al finalizar ( $p$. $<0.05$ ); así como entre las actitudes recogida durante el transcurso del curso y las recogidas al finalizar ( $\mathrm{p}$. < 0.05). En general, se observa como las actitudes hacia la estadística del alumnado no solo se van incrementando a medida que van ocupando un momento distinto del curso, sino que, además, dichos incrementos son significativos. 


\section{Discusiones y Conclusiones}

Se inició este estudio señalando que la estadística en la investigación educativa era una parte integral en la formación de los futuros docentes de Educación Primaria, constituyendo un requerimiento para acercar al aula la innovación docente y consecuentemente, poder conocer la eficacia de los planes y programas que se pongan en práctica. Por ello, el propósito de este estudio era conocer la eficacia de la metodología Flipped Classroom la cual cada vez gana más reconocimiento en una amplia variedad de entornos académicos como enfoque para promover el aprendizaje activo del alumnado, sobre las actitudes hacia la estadística del alumnado del Grado de Educación Primaria, así como identificar si la interacción del sexo provocaba una diferencia significativa.

En relación con la eficacia de la metodología empleada, los resultados en este estudio mostraron diferencias significativas, tanto entre los momentos antes y durante como entre los momentos antes y después, lo que revela la efectividad continua del uso del aula invertida apoyado por la plataforma Blackboard para el desarrollo de las clases virtuales, comunicación con el alumnado y como repositorio de material audiovisual. Estos resultados coinciden con los de otros estudios como los de Wilson (2013), Senger (2016) o Cronhjor et al. (2018), donde la eficacia de esta metodología quedó evidenciada por las diferencias positivas que se hallaron entre los pre-test y post-test desarrollados en los diferentes trabajos.

Apostar por metodologías como el Flipped Classroom en un contexto formativo elearning ha mejorado las actitudes del alumnado respecto al aprendizaje de la Estadística. Este hecho coincide con el trabajo de Mondéjar, Vargas y Mondéjar (2007), donde el alumnado que siguió la asignatura en e-learning redujo su nivel de ansiedad y mejoró la percepción de utilidad hacia esta materia.

Los hallazgos encontrados indican que las actitudes hacia la estadística del alumnado en ambos sexos no presentan diferencias significativas, rechazando la hipótesis nula planteada correspondientemente, lo que contradice los resultados obtenidos en otras investigaciones anteriores como las de Pérez et al. (2015), Estrada et al. (2004), Roca (2008), Chiesi y Primi (2017), y Rosli, Maat y Rosli (2017). Aunque la diferencia no sea muy elevada, aparece que los hombres poseen una puntuación ligeramente superior a las mujeres, cuyos resultados fueron similares a los encontrados por Papanastasiou y Zembylas (2008), Vahedi et al. (2011), Koh y Zawi (2014), y Chowdhury (2018). Estos resultados nos inducen a pensar que el uso de la metodología aula invertida apoyado con sesiones virtuales y repositorio de material audiovisual en Blackboard para aprender estadística, es más positivo para los hombres.

A la vista de los hallazgos encontrados, y a pesar del tamaño de la muestra, nos permite hacer una primera aproximación al papel tan relevante que tienen las actitudes hacia la estadística en los futuros maestros de Educación Primaria, así como contrastar el efecto del sexo sobre las mismas. A partir de este trabajo, se abre un camino para desarrollar trabajos futuros con una muestra superior en la cual aparezca al menos un grupo control y varios grupos experimentales con los que poder mejorar la validez del estudio y consecuentemente, la generalización de los resultados.

Además de este este trabajo futuro, se ha de seguir analizando las interacciones que pueden afectar a las actitudes hacia la estadística de otras variables sociodemográficas o académicas, como, por ejemplo, la edad, las calificaciones que ha marcado la trayectoria académica del estudiante, la implicación del alumno en el grupo de trabajo, su motivación o la frecuencia de participación y asistencia a las clases, entre otras. 


\section{Referencias}

ANASTASIADOU, S. Affective reactions and attitudes of the last class of Greek high school students towards statistics. En BOSCH, M. (Ed.). Proceedings of CERME IV. European Research in Mathematics Education. Girona: ERME, 2005. [CD-ROM].

APARICIO, A.; BAZÁN, J. L. Aspectos afectivos intervinientes en el aprendizaje de la estadística: actitudes y sus formas de evaluación. Acta Latinoamericana de Matemática Educativa, n. 21, p. 180-189, 2008.

ARTEAGA, B.; GARCÍA, M. Diseño y evaluación de estrategias adaptativas para la mejora del rendimiento en matemáticas en educación secundaria. Bordón, v. 62, n. 4, p. 25-35, 2010.

AYCICEK, B.; YELKEN, T. Y. The effect of flipped classroom modelo in students' classroom engagement in teaching English. International journal of instruction, v. 11, n. 2, p. 385-398, 2018. DOI: https://doi.org/10.12973/iji.2018.11226a

BISHOP, J. L.; VERLEGER, M. A. The flipped classroom: A survey of the research. En ASEE National Conference Proceedings, Atlanta, GA, v. 30, n. 9, p. 1-18, 2013.

BLANCO, Á. Una revisión crítica de la investigación sobre las actitudes de los estudiantes universitarios hacia la Estadística. Revista Complutense de Educación, v. 19, n. 2, p. 311330, 2008.

BOND, M. E.; PERKINS, S. N.; RAMIREZ, C. Students' perceptions of Statistics: an exploration of attitudes, conceptualizations, and content knowledge of Statistics. Statistics Education Research Journal, v. 11, n. 2, p. 6-25, 2012.

CAMACHO, X. G. O.; MARTÍNEZ, S. J. R.; DE MIGUEL, C. R. Cuestionario de actitudes hacia la estadística (cahe): evidencias de validez y fiabilidad de las puntuaciones en una muestra de alumnos de educación. Bordón. Revista de pedagogía, v. 68, n. 4, p. 121-136, 2016.

CARLSON, K. A.; WINQUIST, J. R. Evaluating an Active Learning Approach to Teaching Introductory Statistics: A Classroom Workbook Approach. Journal of Statistics Education, v. 19, n.1, 2011. Disponible en: http://www.amstat.org/publications/jse/v19n1/carlson.pdf. Acceso en: 19 oct. 2020.

CARMONA, J. Una revisión de las evidencias de fiabilidad y validez de los cuestionarios de actitudes y ansiedad hacia la Estadística. Statistics Education Research Journal, v. 3, n. 1, p. 5-28, 2004.

CASAS, J. C.; VILLARRAGA, M. E.; MAZ, A.; LEÓN, C. Factores de influencia en las actitudes hacia la estadística de alumnos de educación media. Espacios, v. 39, n. 52, p. 33 [en línea], 2018. Disponible en: http://www.revistaespacios.com/a18v39n52/a18v39n52p33.pdf. Acceso en: 19 oct. 2020.

CHIESI, F.; PRIMI, C. Do attitudes toward statistics change during an introductory statistics 
course? A study on Italian Psychology students. CERME, n. 10, p. 723-731, 2017.

CHOWDHURY, S. K. Prior Knowledge, Sex and Students' Attitude towards Statistics: A Study on Postgraduate Education Students. American Journal of Educational Research, v. 6, n. 3, p. 270-276, 2018.

COMAS, C.; MARTINS, J. A.; NASCIMENTO, M. M.; ESTRADA, A. Estudio de las actitudes hacia la estadística en estudiantes de psicología. Bolema: Boletim de Educação Matemática, v. 31, n. 57, p. 479-496, 2017. DOI: http://dx.doi.org/10.1590/19804415v31n57a23

Cото, M. Descubrimiento del estilo de aprendizaje dominante en estudiantes de Matemática Superior. Revista educación, v. 44, n. 1, 2020. DOI: https://doi.org/10.15517/REVEDU.V44I1.38571

CRONHJORT, M.; FILIPSSON, L.; WEURLANDER, M. Improved engagement and learning in flipped-classroom calculus. Teaching mathematics and its applications, v. 37, $\mathrm{n}$. 3, p. 113-121, 2018. DOI: https://doi.org/10.1093/teamat/hrx007

DARIAS, E. J. Escala de actitudes hacia la estadística. Psicothema, v. 12, n. 2, p. 176178, 2000.

ELIZAR, E.; DARMAWAN, I. G. N. A Multigroup Invariance Analysis and Gender Difference of Students' Self-efficacy and Attitude Concerning Mathematics. Jurnal Didaktik Matematika, v. 5, n. 2, p. 1-10, 2018.

ESCALANTE, E.; REPETTO, A. M.; MATTINELLO, G. Exploración y análisis de la actitud hacia la estadística en alumnos de psicología. Liberabit. Revista de Psicología, v. 18, n. 1, p. 15-26, 2012.

ESPERANZA, P.; FABIAN, K.; TOTO, C. Flipped Classroom Model: Effects on Performance, Attitudes and Perceptions in High School Algebra. En: Verbert, K.; Sharples, M.; Klobucar, T. (Eds.). Adaptive and Adaptable Learning: Lecture Notes in Computer Science. Switzerland: Springer, 2016, v. 9891, p. 85-97. DOI: https://doi.org/10.1007/978$\underline{3-319-45153-4] 7}$

ESTRADA, A.; BAZÁN, J.; APARICIO, A. Evaluación de las propiedades psicométricas de una escala de actitudes hacia la estadística en profesores. Avances de Investigación en Educación Matemática, n. 3, p. 5-23, 2013. DOI: https://doi.org/10.35763/aiem.v0i3.61

ESTRADA, A., BERNABEU, C. B.; AYMEMí, J. M. F. Un estudio comparado de las actitudes hacia la estadística en profesores en formación y en ejercicio. Enseñanza de las ciencias: Revista de investigación y experiencias didácticas, v. 22, n. 2, p. 263-273, 2004.

FROELICH, A. G.; STEPHENSON, W. R.; DUCKWORTH, W. M. Assessment of materials for engaging students in statistical discovery. Journal of Statistics Education, v. 16, n. 2, p. 10-22, 2008. 
GALINDO, H.; BEZANILLA, M. J. Una revisión sistemática de la metodología flipped classroom a nivel universitario en España. Innoeduca. International Journal of Technology and Educational Innovation, v. 5, n. 1, p. 81-90, 2019. DOI: https://doi.org/10.24310/innoeduca.2019.v5i1.4470

GARCÍA, J. A.; FALLAS, M. A.; ROMERO, A. Las actitudes hacia la estadística del estudiantado de orientación. Revista electrónica educare, v. 19, n. 1, p. 25-41, 2015. DOI: http://dx.doi.org/10.15359/ree.19-1.2

GONZÁlEZ, D.; JEONG, J. S.; RODRÍGuEZ, D. A.; CAÑADA, F. Performance and Perception in the Flipped Learning Model: An Initial Approach to Evaluate the Effectiveness of a New Teaching Methodology in a General Science Classroom. Journal of Science Education and Technology, v. 25, n. 3, p. 450-459, 2016. DOI: https://doi.org/10.1007/s10956-016-9605-9

GUÀRDIA, J.; FREIXA, M.; PERÓ, M.; TURBANY, J.; COSCULLUELA, A.; BARRIOS, M.; RIFÀ, X. Factors related to the academic performance of students in the statistics course in Psychology. Quality and Quantity, v. 40, p. 661-674, 2006.

HANNIGAN, A.; GILL, O.; LEAVY, A. M. An investigation of prospective secondary mathematics teachers' conceptual knowledge of and attitudes towards statistics. Journal of Mathematics Teacher Education, v. 16, n. 6, p. 427-449, 2013.

HARVEY, A. L. The Validity of Six Beliefs About Factors Related to Statistics Achievement. En: Annual Meeting of the American Educational Research Association, 69th, 1985, Chicago, IL, March 31-April 4).

IMMEKUS, J. C. Flipping Statistics Courses in Graduate Education: Integration of Cognitive Psychology and Technology. Journal of Statistics Education, v. 27, n. 2, p. 79-89, 2019. DOI: https://doi.org/10.1080/10691898.2019.1629852

JOHNSON, G. B. Student Perceptions of the flipped Classroom. Okaganan: The University of British Columbia, 2013.

KHAVENSON, T.; OREL, E.; TRYAKSHINA, M. Adaptation of survey of attitudes towards statistics (SATS 36) for Russian sample. Procedia-Social and Behavioral Sciences, v. 46, p. 2126-2129, 2012. DOI: http://dx.doi.org/10.1016/j.sbspro.2012.05.440

$\mathrm{KOH}$, D.; ZAWI, M. K. Statistics Anxiety among Postgraduate Students. International Education Studies, v. 7, n. 13, p. 166-174, 2014.

KONG, S. C. Developing information literacy and critical thinking skills through domain knowledge learning in digital classrooms: An experience of practicing flipped classroom strategy. Computers \& Education, v. 78, p. 160-173, 2014. DOI: https://doi.org/10.1016/j.compedu.2014.05.009

LEÓN, C.; CASAS, J. C.; MAZ, A.; VILLARRAGA, M. E. Analysis of attitudinal components towards statistics among students from different academic degrees. PLOS ONE, v. 15, $\mathrm{n}$. 
1, p. 1-13, 2020. DOI: https://doi.org/10.1371/journal.pone.0227213

LIMNIOU, M.; SCHERMBRUCKER, I.; LYONS, M. Traditional and flipped classroom approaches delivered by two different teachers: The student perspective. Education and Information Technologies, v. 23, p. 797-817, 2018. DOI: https://doi.org/10.1007/s10639$\underline{017-9636-8}$

LITTLE, C. The Flipped Classroom in Further Education: Literature Review and Case Study. Research in Post-Compulsory Education, v. 20, n. 3, p. 265-279, 2015.

LÓPEZ, J.; POZO, S.; DEL PINO, M. J. Projection of the Flipped Learning Methodology in the Teaching Staff of Cross-Border Contexts. Journal of New Approaches in Educational Research, v. 8, n. 2, p. 184-200, 2019. DOI: https://doi.org/10.7821/naer.2019.7.431

MARTÍN, D.; TOURÓN, J. El enfoque flipped learning en estudios de magisterio: percepción de los alumnos. RIED. Revista Iberoamericana de Educación a Distancia, v. 20, n. 2, p. 187-211, 2017. DOI: http://dx.doi.org/10.5944/ried.20.2.17704

MASON, G. S.; SHUMAN, T. R.; COOK, K. E. Comparing the Effectiveness of an Inverted Classroom to a Traditional Classroom in an Upper-Division Engineering Course. IEEE Transactions on Education, v. 56, n. 4, p. 430-435, 2013. DOI: https://doi.org/10.1109/TE.2013.2249066

MCBRIDE, C. Flipping Advice for Beginners: What I Learned Flipping Undergraduate Mathematics and Statistics Classes. PRIMUS, v. 25, n. 8, p. 694-712, 2015. DOI: https://doi.org/10.1080/10511970.2015.1031300

MENDAÑA, C.; POY, R.; LÓPEZ, E. Metodología flipped classroom: percepción de los alumnos de diferentes grados universitarios. Innoeduca. International Journal of Technology and Educational innovation, v. 5, n. 2, p. 178-188, 2019. DOI: https://doi.org/10.24310/innoeduca.2019.v5i2.5223

METAUTE, J. C.; VILLARREAL, J. E.; VARGAS, J. P.; SAKER, J.; BUSTAMANTE, L. E. Aula invertida y pedagogía conceptual en la enseñanza y aprendizaje de la estadística en educación superior. El caso de la estimación y la prueba de hipótesis. Espacios, v. 39, n. 10, 2018. Disponible en: https://www.revistaespacios.com/a18v39n10/a18v39n10p39.pdf. Acceso en: 19 oct. 2020.

MONDÉJAR, J.; VARGAS, M.; MONDÉJAR, J. A. Impacto del uso del e-learning en las actitudes hacia la Estadística. Revista Latinoamericana de Tecnología Educativa, v. 6, n. 2, p. 31-47, 2007.

MOTOS, P.; AGUILAR, Á. I.; COLOMO, E.; GABARDA, V. Adquisición en competencias digitales en educación secundaria a través de Flipped Classroom y realidad aumentada. En: López, E.; Cobos, D.; Martín, A. H.; Molina, L.; Jaén, A. (Coords.). Experiencias pedagógicas e innovación educativa: aportaciones desde la praxis docente e investigadora. Barcelona: Octaedro, 2018, p. 2864-2877.

NIELSEN, P. L.; BEAN, N. W.; LARSEN, R. A. A. The Impact of a Flipped Classroom 
Model of Learning on a Large Undergraduate Statistics Class. Statistics Education Research Journal, v. 17, n. 1, p. 121-140, 2018.

O'FLAHERTY, J.; PHILIPHS, C. The use of flipped classrooms in higher education: A scoping review. The Internet and Higher Education, v. 25, p. 85-95, 2015. DOI: https://doi.org/10.1016/j.iheduc.2015.02.002

ORDÓÑEZ, X. G.; ROMERO, S. J.; RUIZ, C. Actitudes hacia la Estadística en Alumnos de Educación: análisis de perfiles. Revista de Educación, n. 385, p. 173-200, 2019. DOI: https://doi.org/10.4438/1988-592X-RE-2019-385-421

PAPANASTASIOU, E. C.; ZEMBYLAS, M. Anxiety in undergraduate research methods courses: Its nature and implications. International Journal of Research \& Method in Education, v. 31, n. 2, p. 155-167, 2008.

PEÑA, A.; SUÁREZ, R.; SANJUÁN, G.; RABELL, O.; GÓMEZ, M.; MORALES, S. C. Actitudes hacia la asignatura de Estadística en estudiantes de la Facultad de Ciencias Médicas "General Calixto García". Revista Habanera de Ciencias Médicas, v. 14, n. 6, p. 872-883, 2015.

PÉREZ, L. E.; APARICIO, A. S.; BAZÁN, J. L.; ABDOUNUR, O. J. Actitudes hacia la estadística de estudiantes universitarios de Colombia. Educación Matemática, v. 27, n. 3, p. 111-149, 2015.

PETERSON, D. J. The Flipped Classroom improves student achievement and course satisfaction in a statistics course: a quasi-experimental study. Teaching of psychology, v. 43, n. 1, p. 10-15, 2016. DOI: https://doi.org/10.1177/0098628315620063

RAMÍREZ, C.; SCHAU, C.; EMMIOGLU, E. The importance of attitudes in statistics education. Statistics Education Research Journal, v. 11, n. 2, p. 57-71, 2012.

ROCA, A. E. Actitudes hacia la estadística: un estudio con profesores de educación primaria en formación y en ejercicio. En: Sociedad Española de Investigación en Educación Matemática (Ed.). Investigación en educación matemática: comunicaciones de los grupos de investigación del XI Simposio de la SEIEM, celebrado en La Laguna del 4 al 7 de septiembre de 2007. España: Sociedad Española de Investigación en Educación Matemática, SEIEM, 2008, p. 121-140.

RODRÍGUEZ, N. Actitudes de los estudiantes hacia la estadística. Interdisciplinaria, v. 28, n. 2, p. 199-205, 2011.

RODRÍGUEZ, N. L.; MONTAÑEZ, E. G.; ROJAS, I. Dificultades en contenidos de Estadística Inferencial en Alumnos Universitarios. Revista Electrónica Iberoamericana de Educación en Ciencia y Tecnología, v. 2, n. 1, p. 57-73, 2010.

ROSLI, M. K.; MAAT, S. M.; ROSLI, R. Students' attitude and anxiety towards statistics: A descriptive analysis. Research on Education and Psychology, v. 1, n. 1, p. 47-56, 2017.

RUIZ DE MIGUEL, C. Actitudes hacia la estadística de los alumnos del Grado de 
Pedagogía, Educación Social y Maestro de Educación Infantil y Maestros de Educación Primaria de la UCM. Educación $X X 1$, v. 18, n. 2, p. 351-374, 2015. DOI: https://doi.org/10.5944/educXX1.12158

SALINAS, J.; MAYÉN, S. A. Estudio exploratorio de las actitudes hacia la estadística en estudiantes mexicanos de bachillerato. Avances de Investigación en Educación Matemática, n. 10, p. 73-90, 2016.

SÁNCHEZ, C.; SÁNCHEZ, M. T.; RUIZ, J. Experiencias reales de aula invertida como estrategia metodológica en la educación universitaria española. Publicaciones, v. 49, n. 2, p. 39-58, 2019. DOI: https://doi.org/10.30827/publicaciones.v49i2.8270

SÁNCHEZ, J.; RUIZ, J.; SÁNCHEZ, E. Flipped classroom. Claves para su puesta en práctica. Edmetic, v. 6, n. 2, p. 336-358. DOI: https://doi.org/10.21071/edmetic.v6i2.5832

SCHWARTZ, T. A. Flipping the Statistics Classroom in Nursing Education. The Journal of Nursing Education, v. 53, n. 4, p. 199-206, 2014. DOI: https://doi.org/10.3928/014843420140325-02

SENGER, E. To FLIP or not to FLIP: Comparative case study in higher education in Turkey. Computers in Human Behavior, v. 64, p. 547-555, 2016. DOI: https://doi.org/10.1016/j.chb.2016.07.034

SHINABERGER, L. Components of a Flipped Classroom Influencing Student Success in an Undergraduate Business Statistics Course. Journal of Statistics Education, v. 25, n. 3, p. 122-130, 2017. DOI: https://doi.org/10.1080/10691898.2017.1381056

SOSA, M. J.; PALAU, R. F. Flipped Classroom para adquirir la competencia digital docente: Una experiencia didáctica en la educación superior. Pixel-Bit. Revista de Medios y Educación, n. 52, p. 37-54, 2018.

STRAYER, J. F. How learning in an inverted classroom influences cooperation, innovation and task orientation. Learning Environ Res, v. 15, p. 171-193, 2012. DOI: https://doi.org/10.1007/s10984-012-9108-4

TAWFIK, A. A.; LILLY, C. Using a flipped classroom approach to support problem-based learning. Technology knowledge and learning, v. 20, n. 3, p. 299-315, 2015. DOI: https://doi.org/10.1007/s10758-015-9262-8

TISHKOVSKAYA, S.; LANCASTER, G. A. Statistical education in the 21st century: A review of challenges, teaching innovations and strategies for reform. Journal of Statistics Education, v. 20, n. 2, p. 1-56, 2012. Disponible en: www.amstat.org/publications/jse/v20n2/tishkovskaya.pdf

TOMÁS, J. M.; LIMA, A.; PACHECO, J. Adaptação e validação transcultural de uma medida de atitudes acerca da estatística. Revista Iberoamericana de Diagnóstico y Evaluación psicológica, v. 1, n. 39, p. 102-112, 2015. 
TOUCHTON, M. Flipping the Classroom and Student Performance in Advanced Statistics: Evidence from a Quasi-Experiment. Journal of Political Science Education, v. 11, n. 1, p. 28-44, 2015.

TOURÓN, J.; SANTIAGO, R. El modelo Flipped Learning y el desarrollo del talento en la escuela. Revista de Educación, n. 368, p. 174-195, 2015. DOl: http://doi.org/10.4438/1988-592X-RE-2015-368-288

TRIANTAFYLLOU, E.; TIMCENKO, O. Introducing a flipped classroom for a statistics course: A Case study. En: Karaoglan, B. (Ed.). Proceedings of 25th Annual Conference European Association for Education in Electrical and Information Engineering (EAEEIE). Francia: EAEEIE, 2014, p. 5-8. Doi: https://doi.org/10.1109/EAEEIE.2014.6879373

VAHEDI, S.; FARROKHI, F.; BEVRANI, H. A Confirmatory Factor Analysis of the Structure of Statistics Anxiety Measure: An examination of four alternative models. Iranian journal of psychiatry, v. 6, n. 3, p. 92-98, 2011.

VANHOOF, S.; KUPPENS, S.; CASTRO, A. E.; VERSHAFFEL, L.; ONGHENA, P. Measuring statistics attitudes: structure of the survey of attitudes towards statistics (SATS36). Statistics Education Research Journal, v. 10, n. 1, p. 35-51, 2011.

VILÀ, R.; RUBIO, M. J. Actitudes hacia la Estadística en el alumnado del grado de Pedagogía de la Universidad de Barcelona. REDU. Revista de Docencia Universitaria, v. 14, n. 1, p. 131-149, 2016.

WILSON, S. G. The flipped class: a method to address the challenges of an undergraduate statistics course. Teaching of psychology, v. 40, n. 3, p. 193-199, 2013. DOI: https://doi.org/10.1177/0098628313487461

WINQUIST, J. R.; CARLSON, K. A. Flipped statitics class results: better perfomance than lecture over one year later. Journal of Statistics Education, v. 22, n. 3, 2014. DOI: https://doi.org/10.1080/10691898.2014.11889717 